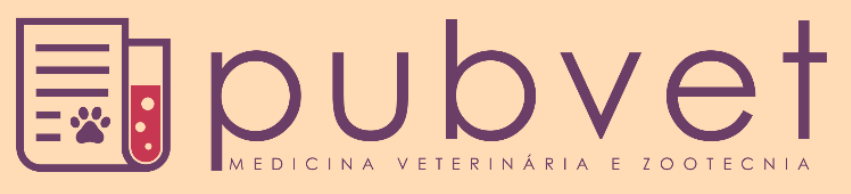

https://doi.org/10.31533/pubvet.v13n6a345.1-5

\title{
Desenvolvimento testicular de touros da raça Sindi
}

\author{
Danillo Velloso Ferreira Murta ${ }^{1 *}$, Daniele Carolina Rodrigues Xavier Murta ${ }^{2}$, Rodrigo Brito de \\ Souza $^{3}$, Cintia Figueiredo de Araújo ${ }^{1}$, Lucélia Karoline Gonçalves Barbosa ${ }^{4}{ }^{\ominus}$, João Marcos Leite \\ Santos $^{50}$, Matheus Gonçalves Costa ${ }^{6}$, Lorena Augusta Marques Fernandes ${ }^{6}$, Patryssa Cardoso \\ Oliveira $^{60}$, Marina Silveira Nonato ${ }^{60}$, Rayane Barbosa Cabral ${ }^{60}$, Anderson Ricardo Reis Queiroz ${ }^{60}$, \\ Antônio Ray Amorim Bezerra ${ }^{6}{ }^{\circ}$, Vitória Cotrim Souza Figueredo ${ }^{6}{ }^{\circ}$, Raquel Geovana Nunes Alves ${ }^{60}$
}

${ }^{1}$ Doutor(a). Docente do Curso de Medicina Veterinária do Centro Universitário UniFG, Guanambi, Bahia, Brasil.

${ }^{2}$ Médica Veterinária Autônoma graduada na Faculdades Integradas do Norte de Minas - Funorte, Montes Claros, Minas Gerais, Brasil

${ }^{3}$ Mestrando. Docente do Curso de Medicina Veterinária do Centro Universitário UniFG, Guanambi, Bahia, Brasil

${ }^{4}$ Mestre. Docente do Curso de Medicina Veterinária do Centro Universitário UniFG, Guanambi, Bahia, Brasil

${ }^{5}$ Mestrando. Departamento de Cirurgia, Faculdade de Medicina Veterinária e Zootecnia, Universidade de São Paulo, Brasil

${ }^{6}$ Acadêmico. Graduando Medicina Veterinária no Centro Universitário UniFG, Guanambi, Bahia, Brasil

*Autor para correspondência, Rua São João - N²79. Bairro: Todos os Santos. Montes Claros, MG. CEP: 39400-126. E-mail: danillo.murta.vet@ gmail.com

Resumo. Nos mamíferos, o crescimento das gônadas está associado à secreção de esteroides e a circunferência escrotal (CE) apresenta correlação significativa com idade à puberdade. Estudos também têm mostrado que as interações entre medidas testiculares, peso corporal e a circunferência escrotal tem sido utilizada em programas de seleção para precocidade sexual. Este estudo objetivou avaliar a evolução da circunferência escrotal em touros da raça Sindi contemporâneo em duas faixas etárias. Utilizou-se um grupo de touros com coletas realizadas em dois tempos. Foram avaliados, em cada momento, o peso corporal, produção espermática e as circunferências escrotais dos indivíduos de cada grupo. Verificou-se que os touros com idade média de 13 meses apresentaram peso médio de $384,67 \mathrm{~kg}$ e circunferência escrotal média de $32,5 \mathrm{~cm}$. Na segunda coleta, os mesmos indivíduos já com idade média de 25 meses apresentaram peso médio de $494 \mathrm{~kg}$ e circunferência escrotal média de $38 \mathrm{~cm}$. Os valores de CE e peso apresentaram diferenças significativas entre os dois grupos nas diferentes faixas etárias. Em função da idade, relacionando-a com o peso corporal e circunferência escrotal dos animais, conclui - se que o perímetro escrotal dos touros Sindi assemelham-se as medidas citadas dos taurinos, que se destacam no quesito precocidade quando comparados as raças zebuínas. Os animais proporcionaram desenvolvimento significativo de peso quando mantidos num sistema especial de confinamento, oferecendo condições para expressar seu potencial genético, capaz de atingir maior peso em menor tempo e iniciar a vida reprodutiva precocemente.

Palavras chave: circunferência escrotal, desenvolvimento ponderal,

\section{Testicular development Sindi bulls}

Abstract. In mammals, the growth of the gonads is associated with the steroids secretion and scrotal circumference (SC), age at puberty significantly correlated. Studies have also shown that the interaction between testicular measurements, body weight and scrotal circumference has been used in breeding programs for sexual precocity. This study aimed to evaluate the evolution of scrotal circumference in Sindi bulls into two contemporaries' age groups. A group of bulls with samples taken at different times was used. In each time, the body weight, sperm output and scrotal circumferences from each bull of the group was evaluated. It was found that the bulls with 13 months average age showed a mean weight of $384.67 \mathrm{~kg}$ and an average scrotal circumference was $32.5 \mathrm{~cm}$. In the second collect, the 
same bulls already with an average age of 25 months had a mean weight of $494 \mathrm{~kg}$ and an average scrotal circumference was $38 \mathrm{~cm}$. The $\mathrm{SC}$ and weight values showed significant differences between different ages in the two groups. Body weight and scrotal circumference of animals depending on age-related, concludes that the scrotal circumference in Sindi bulls resemble those already reported taurine measures, which stand up in the requirement precocity compared to zebu breeds. The animals have provided significant weight development when held in a particular containment system, providing conditions to express their genetic potential, capable of reaching more weight in less time and begin sexual life early.

Keywords: scrotal circumference, weight development

\section{Desarrollo testicular de toros de la raza Sindi}

Resumen. En los mamíferos, el crecimiento de las gónadas está asociado a la secreción de esteroides y la circunferencia escrotal (CE) presenta una correlación significativa con la edad a la pubertad. Los estudios también han demostrado que las interacciones entre las medidas testiculares, el peso corporal y la circunferencia escrotal se ha utilizado en los programas de selección para la precocidad sexual. Este estudio objetivó evaluar la evolución de la circunferencia escrotal en toros de la raza Sindi contemporáneo en dos grupos de edad. Se utilizó un grupo de toros con colectas realizadas en dos tiempos. Se evaluaron, en cada momento, el peso corporal, producción espermática y la circunferencia escrotal de los individuos de cada grupo. Se verificó que los toros con edad media de 13 meses presentaron peso promedio de $384,67 \mathrm{~kg}$ y circunferencia escrotal media de $32,5 \mathrm{~cm}$. En la segunda colecta, los mismos individuos ya con edad media de 25 meses presentaron peso promedio de $494 \mathrm{~kg}$ y circunferencia escrotal media de $38 \mathrm{~cm}$. Los valores de CE y peso presentaron diferencias significativas entre los dos grupos en las diferentes edades. En función de la edad, relacionándola con el peso corporal y circunferencia escrotal de los animales, se concluye que el perímetro escrotal de los toros Sindi se asemejan a las medidas citadas de los taurinos, que se destacan en el aspecto precocidad cuando se comparan con las razas cebuínas. Los animales proporcionaron un desarrollo significativo de peso cuando se mantuvieron en un sistema especial de confinamiento, ofreciendo condiciones para expresar su potencial genético, capaz de alcanzar mayor peso en menor tiempo e iniciar la vida reproductiva precozmente.

Palabra clave: circunferencia escrotal, desarrollo de peso

\section{Introdução}

O Brasil possui atualmente um rebanho bovino de 209 milhões de cabeças. Trata-se do maior rebanho comercial do mundo e mais de 12 milhões de doses de sêmen comercializadas (ANUALPEC, 2018).

A raça Sindi originou do Estado de Sindi no Paquistão (Leite et al., 2001). Sua rusticidade e tolerância ao calor fizeram com que a raça se espalhasse pela Ásia, Oceania, África e Américas. Os animais Sindi são caracterizados pelo pequeno porte e pelagem vermelha, variando de tonalidades de acordo com o sexo do animal. As fêmeas produzem, em média, $1.700 \mathrm{~kg}$ de leite por lactação (Leite et al., 2001). Entretanto, sob condições ótimas, algumas chegam a ultrapassar $4.000 \mathrm{~kg}$ de leite por lactação (Leite et al., 2001). Em 1952 chegaram ao Brasil os primeiros exemplares da raça e, atualmente, a maior parte do rebanho está presente no nordeste brasileiro (Faria et al., 2001; Leite et al., 2001).

O desempenho dos reprodutores depende do aprimoramento de técnicas de manejo e alimentação. Ainda, é fundamental o conhecimento da fisiologia do desenvolvimento testicular e ponderal, além dos fatores que potencialmente interferem estes processos, como a precocidade sexual e a capacidade de produção espermática. Nos bovinos, o crescimento das gônadas está associado à secreção de esteroides e a circunferência escrotal (CE) apresenta correlação significativa com idade à puberdade (Lunstra et al., 1978). 
Fêmeas de raças cujos touros têm CE mais desenvolvido possuem melhores índices de fertilidade e são mais precoces (Martin et al., 1992; Martins Filho \& Lôbo, 1991), sugerindo que medidas testiculares constituem parâmetros que podem auxiliar no processo de melhoria da eficiência reprodutiva dos rebanhos. Estudos também têm mostrado que as interações entre medidas testiculares, peso corporal e a circunferência escrotal tem sido utilizada em programas de seleção para precocidade sexual (Garnero et al., 1999).

Objetivou-se descrever a circunferência escrotal de touros jovens da raça Sindi com idade média entre 13 e 25 meses, comparando as medidas de circunferência escrotal com outras raças zebuínas e taurinas relatados na literatura.

\section{Material e métodos}

Utilizamos um grupo de touros da raça Sindi da Fazenda Barra da Vereda, no município de São João da Ponte no Norte de Minas Gerais, que possui clima semiárido, caracterizado por altas temperaturas e baixa umidade. As coletas de dados foram realizadas em dois tempos, conforme a idade dos animais. Foi avaliado em cada momento, o peso corporal em balança eletrônica individual e mensuradas as circunferências escrotais dos indivíduos de cada grupo com uma fita métrica, no seu local de maior largura, envolvendo as duas gônadas e a pele escrotal. Realizado também, exame andrológico com uso de eletro ejaculador, verificando a produção espermática, para descrever o período em que os animais atingiram a puberdade.

Os animais permaneceram durante aproximadamente seis meses (abril - outubro; 168 dias) em sistema de confinamento para realização da prova de ganho de peso da Raça Sindi, realizada na propriedade, com dieta a base de silagem de milho, sendo que a primeira coleta de dados foi realizada ao final deste período. Em seguida, os animais foram mantidos em sistema de criação extensivo, a pasto da gramínea Brachiaria brizantha, e suplementação mineral, passando por um processo de readaptação. A segunda coleta de dados foi realizada em junho (oito meses depois), sendo repetidas as mesmas análises.

Avaliou-se o desenvolvimento testicular em função das diferentes idades, por meio da análise de variância e do teste de Duncan $(\mathrm{P}<0,05)$.

\section{Resultados e discussão}

Os resultados referentes ao peso corporal e circunferência escrotal dos touros da raça Sindi estão demonstradas na tabela 1. Verificou-se que os touros com idade média de 13 meses apresentaram peso médio de $384,7 \mathrm{~kg}$ e circunferência escrotal média de $32,5 \mathrm{~cm}$. Na segunda coleta, os mesmos indivíduos já com idade média de 25 meses apresentaram peso médio de $494 \mathrm{~kg}$ e circunferência escrotal média de $38 \mathrm{~cm}$. Os valores de CE e peso apresentaram diferenças entre os dois grupos em diferentes faixas etárias.

A circunferência escrotal é relacionada à produção espermática, peso dos testículos e idade à puberdade (Pinho et al., 2001). Estudando a biometria testicular de zebuínos, Neves (2007) encontraram em touros da raça Nelore criados a pasto, com idade de 24,9 meses valor média para circunferência escrotal de 27,7 cm. Estudos de Unanian et al. (2000), com 442 touros da raça Nelore, evidenciaram que aos 12 e 18 meses apresentaram respectivamente circunferência escrotal de 21,43 e 22,01 cm. Enquanto touros da raça Guzerá, criados a pasto na região do cerrado, recebendo suplementação volumosa no período da seca, foram avaliados aos 22 meses de idade e apresentaram perímetro escrotal de $25,7 \mathrm{~cm}$ e peso corporal de $274,5 \mathrm{~kg}$ (Osorio et al., 2012). Todos os relatos citados para raças zebuínas foram inferiores aos identificados no presente estudo em touros da raça Sindi em idade aproximadas, o que revela a alta precocidade sexual da raça. Brito et al. (2004) registraram idades de 20 e 23,8 meses para puberdade e maturidade sexual na raça Nelore, respectivamente.

Tabela 1. Peso e desenvolvimento testicular em touros da raça Sindi em diferentes faixas etárias

\begin{tabular}{lcc}
\hline Idade média, meses & Circunferência escrotal, cm & Peso, $\mathrm{kg}$ \\
\hline 13 & $32,5^{\mathrm{a}}$ & $384,67^{\mathrm{a}}$ \\
25 & $38,0^{\mathrm{b}}$ & $494,00^{\mathrm{b}}$ \\
Coeficiente de variação, \% & 5,69 & 3,04 \\
\hline$P<0,05$ & &
\end{tabular}


A idade ao primeiro parto é característica de grande importância zootécnica, marcando o início do processo produtivo das fêmeas. A redução da idade ao primeiro parto antecipa a idade produtiva, provoca rápida recuperação do investimento, aumenta a vida útil, possibilita maior intensidade de seleção nas fêmeas e reduz o intervalo entre gerações (Notter, 2012). A seleção de touros com maior CE e características de crescimento, também vai afetar positivamente a fertilidade das fêmeas, melhorando a eficiência reprodutiva das mesmas (Kriese et al., 1991). O perímetro escrotal, em animais jovens, é um indicador útil do tamanho testicular, da capacidade de produção espermática, das características físicas do sêmen, da idade à puberdade e fertilidade dos machos, bem como das fêmeas aparentadas com estes animais (Peña et al., 2001).

Em trabalhos com touros taurinos da raça Bonsmara aos 25 meses apresentou circunferência escrotal de $37 \mathrm{~cm}$ (Scheepers et al., 2010), touros da raça Devon, Red Angus e Polled Hereford, todos com idade média de 36 meses, obtiveram circunferência escrotal de $38,06 \mathrm{~cm}, 37,88 \mathrm{~cm}$ e $37,86 \mathrm{~cm}$, respectivamente (Menegassi et al., 2011), touros da raça Aberdeen Angus aos 48 meses revelaram circunferência escrotal média de 37,2 cm (Gottschall \& Mattos, 1997).

Estudos de Rossi et al. (2009) realizados com tourinhos do composto Red Norte (Nelore x Tabapuã x Red Angus x Senepol) apontam animais aos 14 meses de idade com peso de $427 \mathrm{~kg}$ e CE de 34,6 cm.

$\mathrm{Na}$ avaliação andrológica verificou-se que aos 13 meses $50 \%$ dos animais apresentaram produção espermática com motilidade acima de $60 \%$, demonstrando que estes atingiram a puberdade nesta faixa etária. Na segunda coleta aos 25 meses, $100 \%$ dos animais já produziam sêmen.

O perímetro escrotal (PE) é utilizado como critério de seleção por ter correlação genética favorável com características de qualidade do sêmen, com a idade à puberdade em machos e fêmeas, e características de crescimento (Fonseca, 2000). Além disso, apresenta facilidade de medição e alta herdabilidade, qualidades desejáveis em uma ferramenta a ser utilizada como critério de seleção (Pereira et al., 2000).

\section{Conclusão}

Touros da raça Sindi apresentaram maior desenvolvimento testicular e corporal, além da produção espermática, demonstrando maior precocidade e menor idade púbere que outras raças zebuínas e assemelhando-se aos taurinos e seus cruzamentos. Os animais proporcionaram desenvolvimento significativo de peso quando mantidos num sistema especial de confinamento, oferecendo condições para expressar seu potencial genético, capaz de atingir maior peso em menor tempo e iniciar a vida reprodutiva precocemente.

A raça Sindi não está mais em processo de formação, sendo capaz de superar outros grupamentos étnicos zebuínos existentes no Brasil, levando à recomendação para uso em determinadas situações, como por exemplo, produção econômica de leite e carne no Semiárido. Isto é decorrente de um melhor desempenho, quando criada sob condições adversas de clima, sendo capaz de produzir e de se reproduzir em ambientes considerados inadequados para outras raças.

\section{Referências bibliográficas}

ANUALPEC. (2018). Anuário da Pecuária Brasileira (20th ed. Vol. 1). São Paulo, São Paulo, Brasil: Instituto FNP.

Brito, L. F. C., Silva, A. E. D. F., Unanian, M. M., Dode, M. A. N., Barbosa, R. T. \& Kastelic, J. P. (2004). Sexual development in early-and late-maturing Bos indicus and Bos indicus $\times$ Bos taurus crossbred bulls in Brazil. Theriogenology, 62(7):1198-1217.

Faria, F. J. C., Vercesi Filho, A. E., Madalena, F. E. \& Josahkian, L. A. (2001). Parâmetros populacionais do rebanho Sindi registrado no Brasil. Revista Brasileira de Zootecnia, 30(6):19891994.

Fonseca, V. O. (2000). O touro no contexto da eficiência reprodutiva do rebanho. Informe Agropecuário, 21(205):48-63. 
Garnero, A. V., Lôbo, R. B. \& Oliveira, H. N. (1999). Estimativas de componentes de variância e parâmetros genéticos de características alternativas na raça Nelore. Reunião Anual da Sociedade Brasileira de Zootecncia, 1166.

Gottschall, C. S. \& Mattos, R. C. (1997). Achados de exames andrológicos em touros de corte Bos taurus e Bos indicus. Revista Brasileira de Reprodução Animal, 21(4):25-28.

Kriese, L. A., Bertrand, J. K. \& Benyshek, L. L. (1991). Age adjustment factors, heritabilities and genetic correlations for scrotal circumference and related growth traits in Hereford and Brangus bulls. Journal of Animal Science, 69(2):478-489.

Leite, P. M., Santiago, A., Navarro Filho, H., Albuquerque, R. \& Leite, R. (2001). Sindi: Gado vermelho para o semi-árido: EMEPA-PB; Banco do Nordeste.

Lunstra, D. D., Ford, J. J. \& Echternkamp, S. E. (1978). Puberty in beef bulls: hormone concentrations, growth, testicular development, sperm production and sexual aggressiveness in bulls of different breeds. Journal of Animal Science, 46(4):1054-1062.

Martin, L. C., Brinks, J. S., Bourdon, R. M. \& Cundiff, L. V. (1992). Genetic effects on beef heifer puberty and subsequent reproduction. Journal of Animal Science, 70(12):4006-4017.

Martins Filho, R. \& Lôbo, R. B. (1991). Estimates of genetic correlations between sire scrotal circumference and off springage at first calving in Nelore cattle (short communication). Revista Brasileira de Genética, 14(1):209-212.

Menegassi, S. R., Barcellos, J. O., Peripolli, V., Pereira, P. R. R., Borges, J. B. \& Lampert, V. N. (2011). Determinação da circunferência escrotal em touros de corte no Rio Grande do Sul. Arquivo Brasileiro de Medecina Veterinária e Zootecnia, 63(1):87-93.

Neves, A. L. A. (2007). Biometria e morfologia testicular em bovinos da raça Nelore criados a pasto. Master of Science, Universidade Estadual do Sudoeste da Bahia, Itapetinga.

Notter, D. R. (2012). Genetic improvement of reproductive efficiency of sheep and goats. Animal Reproduction Science, 130(3-4):147-151.

Osorio, J. P., Henry, M., Bergmann, J. A. G., Carmo, A. S. \& Souza, F. A. (2012). Desenvolvimento testicular e puberdade em machos da raça guzerá da desmama aos 36 meses de idade criados no cerrado mineiro. Revista de Medicina Veterinaria, 249-24.

Peña, C. D. O., Queiroz, S. A. \& Fries, L. A. (2001). Comparação entre critérios de seleção de precocidade sexual e a associação destes com características de crescimento em bovinos Nelore. Revista Brasileira de Zootecnia, 3093-100.

Pereira, E., Eler, J. P. \& Ferraz, J. B. S. (2000). Correlação genética entre perímetro escrotal e algumas características reprodutivas na raça Nelore. Revista Brasileira de Zootecnia, 29(6):1676-1683.

Pinho, T. G., Nogueira, L. A. G., Pinto, P. A., Zamborlini, L., Gilardi, S., Caldas, M. \& Souza, R. M. (2001). Características seminais de touros jovens nelore (Bos taurus indicus) de acordo com a biometria e morfologia testicular. Revista Brasileira Reprodução Animal, 25(2):187-189.

Rossi, R. O. D., Barreto Filho, J. B., Carvalho, P. H., Lopes, E. \& Gonçalvez, T. M. (2009). Características andrológicas e do sêmen de touros do composto Red Norte (Nelore x Tabapuã x Red Angus x Sinepol). Arquivo Brasileiro de Medecina Veterinária e Zootecnia, 61(6):1297-1301.

Scheepers, S. M., Annandale, C. H. \& Webb, E. C. (2010). Relationship between production characteristics and breeding potential of 25-month old extensively managed Bonsmara bulls. South African Journal of Animal Science, 40(3):163-172.

Unanian, M. M., Silva, A. E. D. F., Pimentel, C. M. M. \& Cardoso, E. P. (2000). Características biométricas testiculares para avaliação de touros zebuínos da raça Nelore. Revista Brasileira de Zootecnia, 29136-144.

Recebido: 30 de abril, 2019.

Aprovado: 28 de maio, 2019

Publicado: 26 de junho, 2019.

Licenciamento: Este artigo é publicado na modalidade Acesso Aberto sob a licença Creative Commons Atribuição 4.0 (CC-BY 4.0), a qual permite uso irrestrito, distribuição, reprodução em qualquer meio, desde que o autor e a fonte sejam devidamente creditados. 